\title{
INSTABILITY THROUGH ANISOTROPY IN SPHERICAL STELLAR SYSTEMS
}

\author{
P.L. Palmer and J. Papaloizou \\ School of Mathematics \\ Queen Mary College \\ Mile End Road, London E1 4NS \\ United Kingdom
}

\begin{abstract}
We consider the linear stability of spherical stellar systems by solving the Vlasov and Poisson equations which yield a matrix eigenvalue problem to determine the growth rate. We consider this for purely growing modes in the limit of vanishing growth rate. We show that a large class of anisotropic models are unstable and derive growth rates for the particular example of generalized polytropic models. We present a simple method for testing the stability of general anisotropic models. Our anlysis shows that instability occurs even when the degree of anisotropy is very slight.
\end{abstract}

\section{THE EIGENVALUE PROBLEM AND STABILITY TEST}

In the limit of low growth rate, $\sigma$, the radial orbit resonance, $2 \Omega_{\theta}=\Omega_{r}$, plays an important role. Here $\Omega_{\theta}$ and $\Omega_{r}$ are the angular and radial orbital frequencies. If for small $J, f(E, J) \alpha J^{-s}$, then as $\sigma \rightarrow 0$ the eigenvalue problem reduces to

$$
\frac{-4 \pi G}{\sigma^{s}} \int K\left(r, r^{1}\right) \psi^{1}\left(r^{1}\right) d r^{1}=\frac{1}{r^{2}} \frac{d}{d r}\left[r^{2}\left[\frac{d \psi^{1}}{d r}\right]\right]-n(n+1) \psi^{1} / r^{2}
$$

where $\psi^{1}$ is the perturbed gravitational potential, $n$ the order of the associated spherical harmonic, and the kernel $K$ is positive definite. Further details will be published elsewhere. Equation (1) has a low growth rate spectrum with accumulation point at zero for any $s$. A similar result occurs for any $f$ such that $f \rightarrow \infty$ for $J \rightarrow 0$. We have also been able to derive an equation analogous to (1) which can decide stability for more general $f(E, J)$, (more details to be published elsewhere).

We have calculated the low growth rate spectrum for some generalized polytropes, with $f \propto\left(E_{0}-E\right)^{\beta} J^{-s}$ for $E \leq E_{0}$, and $f=0$ otherwise. These models were studied numerically by Barnes et al. (1986), and Fridman and Polychenko (1984). However, stability boundaries were not well determined in their work. 


\section{NATURE OF THE INSTABILITY}

For nearly radial orbits $\Omega_{r}<2 \Omega_{e}$, so they precess slowly in the direction of orbital rotation. A bar-like perturbation attracts such an orbit and increases its angular momentum as it does so. The orbit then passes through the bar with an angular momentum greater than it had initially if it started almost radial. If the orbit can reinforce the bar as it passes through, the bar is then able to attract and trap orbits with even lower initial angular momentum, which replace those already trapped but which have moved to higher angular momentum. From this it follows that we expect instability when $d f / d J<0$.

A similar mechanism has been proposed by Lynden-Bell (1979) for the formation of bars in disc galaxies.

\section{REFERENCES}

Lynden-Bell, D., 1979 Mon. Not. R. astr. Soc., 187, 101.

Barnes, J., Goodman, J., and Hut, P., 1986, Astrophys. J., 300, 112.

Fridman, A.M., and Polyachenko, V.L., 1984, Physics of Gravitating Systems I, Chap. 3, Springer-Verlag, New York. 